Conclusions: Second-year students have sufficient background to understand molecular pathology techniques and are interested in learning diagnostic and molecular pathology. The case-based format fostered mastery of the material and student-run presentations integrated the video data, literature resources and other data students had gathered independently. The initial pilot was well received. The lower response score regarding clinical decision-making (3.6) likely reflects the emphasis of diagnostic and molecular pathology in the pilot. Future sessions will provide data for improving the structure and content of this model of teaching and its integration into the medical curriculum.

\section{Electron Microscopy}

\section{OSMOPROTECTANTS IN TISSUE SAMPLES PROCESSED FOR IMMUNOGOLD ELECTRON MICROSCOPY}

Mark Koina, ACT Pathology, The Canberra Hospital, Garran, Australia; Lesley Maxwell, ACT Pathology, Garran, Australia; Jane Dahlstrom, ACT Pathology, The Canberra Hospital and Australian National University Medical School, Garran, Australia Background: Osmoprotectants are naturally occurring chemicals that are accumulated in stress tolerant organisms and serve to protect membranes and/or proteins. Previously we have demonstrated that some osmoprotectants can protect the ultrastructure of cell suspensions in LR-white, a traditional immunogold labelling resin. The aims of this study were to examine the effect of adding certain osmoprotectants to the primary electron microscopic fixative and assessing its impact on immunogold labelling of these tissue samples and ultrastructural preservation following processing into LR White and epoxy resins.

Design: Samples of pancreas were primary fixed in $2 \%$ Glutaraldehyde with various osmoprotectants including Betaine, Carnitine, Ectoine and Methyl Gluco-pyronoside. The tissues were treated with various antigen retrieval methods and subsequently processed into LR-white or epoxy resins in the standard way. Immunogold labelling was performed and the quality of the labelling and preservation of ultrastructure assessed independently by two electron microscopists.

Results: Carnitine, Ectoine and Methyl Gluco-pyronoside resulted in improved preservation of the tissues and improved immunogold labelling. The antibody was easier to localise making interpretation simpler.

Conclusions: The addition of certain osmoprotectants in the processing of tissue for electron microscopic examination leads not only to better preservation of the tissue but also improved immunogold labelling efficiency and easier identification of antigenic sites.

\section{CELLULAR ANGIOFIBROMA OF THE CORPUS SPONGIOSUM: CASE REPORT}

Lorena Posligua, Baylor College of Medicine, Houston, TX, United States; Ittmann Michael; Krishnan Bhuvaneswari, Baylor School of Medicine/Michael E DE Bakey VA Medical Center, Houston, TX, United States

Background: Cellular angiofibroma (CA) is a benign mesenchymal neoplasm. It was initially described, arising in the vulva by Nucci et al in 1997. We report a case of cellular angiofibroma arising in the bulbar corpus spongiosum alone with the electron microscopic findings.

Clinical History: The patient is a 65 year old white male who presented with a slow growing, lobulated, well circumscribed $6.5 \times 5.7 \times 3.4 \mathrm{~cm}$ mass arising from the corpus spongiosum in the penile bulb. Gross Findings: The specimen consists of and irregular piece of tan-white soft tissue that measured $7.0 \times 5.0 \times 2.5 \mathrm{~cm}$ with a stalk-like portion that measures $2.0 \times 2.0 \times 0.2 \mathrm{~cm}$.

Results: The tumor consisted of bland spindle cell proliferation in a edematous fibrous stroma with fine wispy collagen, with multiple small to medium sized blood vessels. The spindle cell proliferation was evenly distributed without hypo or hypercellular areas or clustering around the vessels. Mast cells were evenly distributed throughout the tumor. The tumor cells showed strong positivity for CD34, diffuse, moderate staining for desmin, focal and moderate staining for smooth muscle actin. Nuclear staining for estrogen receptor was seen in most of the spindle cells and rare spindle cell nucleus showed staining for progesterone receptors. Electron Microscopic Findings: The spindled tumoral cells were in a edematous collagenous stroma. The cells were evenly distributed with absence of cell junctions. The spindle cells showed no basal lamina lining the cells. The cell borders were irregular with a few subplasmalemmal densities. No definitive cell to stroma attachment sites were seen. The cytoplasm showed prominent dilated rough endoplasmic reticulum. Golgi complexes were also seen. Microfilaments were arranged along the long axis of the cytoplasm, but no densities were seen. No pinocytotic vesicles were present

Conclusion: Primary soft tissue tumors of the penis are rare. In the current classification of soft tissue tumors by the World Health Organization, Cellular angiofibroma also encompasses tumors previously described as angiomyofibroblastoma-like tumors described in males. Cellular angiofibroma in males has been previously described in the scrotal-inguinal and perineal region. Other rare locations in males are the anus, testis, lumbar and mid trunk soft tissue. In the largest series of 51 cases by Iwasa et al, there were no tumors arising from the corpus spongiosum. Cellular Angiofibroma shows an immunophenotype that suggests fibroblast/myofibroblast as the cell of origin. In the study by Iwasa CA showed positivity for CD34 in $75 \%$, SMA in $25 \%$, and desmin in $8 \%$ of the male cases. 5 The electron microscopic features of this tumor also confirm that impression. These cells showed predominantly fibroblastic features with the absence of a basal lamina, prominent dilated rough endoplasmic reticulum, golgi complexes and microfilaments. Subplasmalemmal densities and irregular cell contours favored a myofibroblastic feature. No cell to stromal attachment sites were seen. The differential diagnosis to be considered baseb on the location of the tumor should include angiomyofibroblastoma, myointimal proliferation, angiocentric myofibroblastic tumor and perineal nodular induration.
203 APICAL CYTOPLASMIC SEGREGATION: A PECULIAR ULTRASTRUCTURAL FEATURE FOUND IN METANEPHRIC ADENOMA

Masayuki Shintaku, Osaka Red Cross Hospital, Osaka, Japan

Background: In a typical case of metanephric adenoma of the kidney, a peculiar ultrastructural feature was found in the apical cytoplasm of neoplastic cells.

Design: Electron microscopic case examination.

Results: The tumor, measuring about $1.5 \mathrm{~cm}$ in diameter, was incidentally found in the righ kidney of a 40-year-old woman. It consisted of a dense proliferation of small cuboidal cells forming round tiny tubules, and the apical portion of the cytoplasm exhibited a club-like swelling and protruded into the lumen. Cytoplasmic organelles were poorly developed in this portion, and it was largely occupied by free ribosomes. Cytoplasmic debris containing the same substances floated in the lumen. The morphological features resembled apocrine (decapitation) secretion, but no secretory granules were found.

Conclusion: This finding probably indicates a process in which the disused portion of the cytoplasm is segregated and abandoned into the lumen, and is different from the commonly seen apoptotic or autodigestive processes. This finding, which is termed tentatively the 'apical cytoplasmic segregation', is probably not a finding specific for this neoplasm but a more universal phenomenon, although it has not been well described, heretofore.

\section{Endocrine}

\section{EVALUATION OF DIABETIC MACRO-AND MICROANGIOPATHIES BY DIGITAL THERMOGRAPHY}

Vasile Anestiadi; Vasile V. Anestiadi, Centre for Pathobiology and Pathology, Academy of Sciences, Chisinau, Moldova; Zinaida G., Anestiadi, Chair of Endocrinology, Medica University, Chisinau, Moldova; Ilie T. Tsiple, Centre for Pathobiology and Pathology, Academy of Sciences, Chisinau, Moldova; Ion V. Cosciug, Medical University, Chisinau, Moldova

Background: The objective of this study was to determine the potential value of digital thermography (DT) for the evaluation of macro- and microangiopathies of lower limbs in diabetes mellitus (DM)

Design: We have studied 250 pts with DM, of which 120 male. 100 pts had DM type I, 150 pts had DM type II, with age varying from 27 to $80 \mathrm{yrs}$, mean age (mean \pm SEM) was $56.2 \pm 6.3$ years. Thermograms were acquired at a constant ambient temperature, in fasting state, using an original PC-interfaced thermograph. 2000x150 thermal values were acquired for each study, with a 10-bit depth, subsequently averaged and reformatted to $128 \times 128$ square 8-bit images. Analysis included noise and horizontal band filtering of raw data, 14\% background cutoff, nine-point kernel image filtering, obtaining $\mathrm{x}$ - and $\mathrm{y}$-axis profiles and averaged temperature curves (ATC), determination of local maximums and minimums of the ATCs, definition of anatomic regions, building of regions of interest (ROI), ROI histograms, viewing in 7 color tables.

Results: Five main thermogram patterns were found: 1 . asymmetric unilateral hypothermia (delta- $t=0.3$ grades Celsius) of 1 st toe (in $35 \%$ of DM I cases); 2 . bilateral symmetrical hypothermia of both feet at the level of distal phalanges (in $25 \%$ of DM I cases); 3 unilateral hypothermia of foot ('thermo-amputation'); 4. unilateral hyperthermia of foot and tibial region; 5. focal hyperthermic regions (diameter from 2 to $4 \mathrm{~cm}$.; indicating potential subsequent ulceration). ATCs and symmetric left-right ROI comparisons helped distinguishing between macro- and microvascular disease, between neuro- and angiopathy in DM, evaluating therapy effectiveness. DT was most valuable in surgical cases, permitting (1) to locate the optimum level of amputation and (2) to detect early the inflammation. Conclusion: DT results in DM correlate well with pathomorphology, and are influenced by DM type, DM duration, and presence of local inflammation.

\section{PERSISTENT HYPERINSULINEMIC HYPOGLYCEMIA AND} NESIDIOBLASTOSIS AFTER BARIATRIC SURGERY

Erin Brooks; Maureen Harmon; Thomas Trainer, University of Vermont/Fletcher Allen Health Care, Burlington, VT, United States

Background: Persistent hyperinsulinemic hypoglycemia in adults is most commonly attributable to insulinomas. Recently, there have been increasing numbers of patients with radiologic findings negative for insulinoma whose symptoms have improved after partial pancreatic resection. Resected specimens reveal beta cell hypertrophy, increased ductuloinsular complexes, and increased size and/or number of islets-features consistent with nesidioblastosis.

Design: Case report of persistent hyperinsulinemic hypoglycemia with nesidioblastosis post gastric-bypass surgery.

Results: A 44 year old woman with a medical history significant for Roux-en-Y gastric bypass surgery in 1994 and erratic blood sugars presented to the endocrinology clinic. Laboratory studies revealed hypoglycemia, hyperinsulinemia, and elevated c-peptide (glucose $48 \mathrm{mg} / \mathrm{dL}$, insulin $5.5 \mathrm{uIU} / \mathrm{ml}$, and C-peptide $2.8 \mathrm{ng} / \mathrm{mL}$ ). Abdominal CT revealed no evidence of insulinoma. A calcium stimulation test with sampling of the splenic artery revealed a doubling of insulin levels, suggestive of nesidioblastosis. The patient's C-peptide was also elevated at this time, indicating a hyperinsulinemic state not attributable to exogenous insulin use. Distal pancreatectomy was performed. Gross examination revealed a $11.5 \times 3.5 \times 1.5 \mathrm{~cm}$ tan tissue fragment with no discrete nodules or masses. Microscopic examination revealed foci of prominent ductule-islet complexes as well as focal hyperplasia of islets with minimal nuclear enlargement. Focal mucinous metaplasia of small interlobular ducts was also identified.

Conclusion: Nesidioblastosis is an extremely rare condition that almost never develops in adulthood. We present the case of a woman who was status-post Roux-en-Y gastric bypass, who subsequently developed persistent hyperinsulinemic hypoglycemia and nesidioblastosis. At her one month follow-up after distal pancreatectomy, she reported no further symptomatic episodes of hypoglycemia. Blood sugars were checked $4-6$ times per day, with a fasting blood sugar range of $80-90 \mathrm{mg} / \mathrm{dL}$. The frequency of nesidioblastosis 
after bariatric surgery has been shown to markedly exceed that of the general population: some studies report a 400 -fold increased frequency. While a direct causal link between morbid obesity, gastric bypass, and impaired function of pancreatic beta cells remains to be elucidated, there has been data implicating glucagon-like peptide 1 (GLP-1) in the pathogenesis. GLP-1 is secreted by enteroendocrine intestinal L cells, and has been shown to increase islet proliferation and neogenesis, as well as decrease islet cell apoptosis. Other contributory factors may include decreased ghrelin levels or post-bypass adaptive changes in patients with pre-existing obesity-related insulin resistance. As the obesity epidemic and number of bariatric surgeries increase, the incidence of adult nesidioblastosis and hyperinsulinemic hypoglycemia is likely to increase as well. Further studies are necessary to clarify the precise cause-and-effect mechanism.

\section{E-CADHERIN EXPRESSION AND CLINICOPATHOLOGIC FEATURES OF PAPILLARY THYROID CARCINOMA ASSOCIATED WITH HASHIMOTO'S THYROIDITIS}

Fernando Candanedo-Gonzalez; Armando Gamboa-Dominguez, Instituto Nacional de Ciencias Medicas y Nutricion, Mexico City, Mexico

Background: E-cadherin and epidermal growth factor receptor (EGFR) expression has been associated with prognostic impact in differentiated thyroid tumors. To determine a relation between Hashimoto's thyroiditis (HT) associated differentiated papillary thyroid carcinoma (D-PTC) and their association with clinical stage, recurrence, E-cadherin and EGFR expression.

Design: An exploratory study was carried out including women of any age with D-PTC with and without evidence of HT. Tissue microarray slides stained for E-cadherin (Dako, clone NH-38) and EGF-R (EGFRpharmDx, Dako) were blindly reviewed by two pathologists. Cases were evaluated according AJCC system and reclassified using WHO categories. Student $t$ test, Fisher test and odd ratio (OR) were used to evaluate associations.

Results: 215 patients with D-PTC were included in the study and $16(7 \%)$ were associated with HT. Mean age was $33.31+10.79$ years for patients with HT versus $40.41+14.7$ years for control group $(\mathrm{p}=0.023)$. HT was associated with multi centricity $(\mathrm{p}=0.016$; $\mathrm{OR}=4.071, \mathrm{CI}=1.269-13.038)$, stages I-II $(\mathrm{p}=0.044 ; \mathrm{OR}=0.141, \mathrm{CI}=0.018-1.088)$, pT1-2 $(\mathrm{p}=0.025 ; \mathrm{OR}=0.025, \mathrm{CI}=0.108-0.857)$, no extra thyroid invasion $(\mathrm{p}=0.002 ; \mathrm{OR}=0.173$, $\mathrm{CI}=0.054-0.557)$ and low E-cadherin expression $(\mathrm{p}=0.018 ; \mathrm{OR}=0.275, \mathrm{CI}=0.098-0.776)$. Recurrences were observed in $13 \%$ vs $14 \%$ patients with and without TH. Mean follow up for all patients was 70 months (range 0-234 months). There was no association with EGF-R expression.

Conclusion: Patients with D-PTC and HT had multi centric intra thyroid tumors in early stages and with reduced E-cadherin expression.

\section{A PATHOLOGICAL REVIEW OF 13 CASES OF ADRENOCORTICAL CARCINOMA}

Muhammad Shafiq Gill; Sarah Johnson, Dept of cellular pathology, Royal Victoria Infirmary, Newcastle upon Tyne, United Kingdom

Background: Adrenocortical carcinoma is a rare tumor afflicting only one to two persons per one million population. It usually occurs in adults. Discerning malignancy in resected adrenocortical neoplasms and predicting future behaviour can be problematic for histopathologists. Various combinations of criteria have been proposed to address this (14).The Royal College of Pathologists (RCPath), United Kingdom, has recently produced a minimum dataset for reporting of adrenocortical neoplasms based mainly on Weiss criteria (5). This study aimed to review cases of adrenocortical carcinoma diagnosed in our department during a ten year period (1996-2005).

Design: Thirteen cases of adrenocortical carcinoma were retrieved and reports were reviewed against the RCPath dataset. Slides were reviewed blind to the original microscopic report.

Results: There were 8 female and 5 male patients. The age ranged from 29 to 81 years (median 50 years). Macroscopically, specimen weights ranged from 21-1655g (mean $226 \mathrm{~g}$, median $250 \mathrm{~g}$ ). The tumour size ranged from $30-190 \mathrm{~mm}$ (mean $116 \mathrm{~mm}$ ). An intact adrenal capsule was seen in 3 out of 9 cases. The table shows adequacy of reporting of the microscopic criteria in the original reports and their comparison with findings on subsequent review. Overall, one case showed 3 of the criteria suggestive of malignancy, ten cases showed 5-8 criteria while two cases showed 9 or above criteria. Microscopic Features Presence or absence of findings noted in the original report (13 cases) vs Positive findings on review (11 cases)

$>1 / 3$ diffuse architecture----------------10 (77\%)-----11(100\%)

$<25 \%$ clear cells----------- $3(23 \%)---11(100 \%)$

Significant nuclear pleopmorphism---12 (92\%)------6 (55\%)

Confluent necrosis------------------ $9(69 \%)----10(91 \%)$

Mitoses $\geq 6$ per 50 HPF (x400)-------10 $(77 \%)-----9(82 \%)$

Abnormal mitoses------------------ 7 (54\%)-----8 (73\%)

Broad fibrous bands--------------------2 (15\%)-----6 (55\%)

Capsular invasion-------------------13 (100\%)----6 (55\%)

Venous invasion--------------------------12 (92\%) -----6 (55\%)

Sinusoidal invasion------------------------6 (46\%)-----0 (0\%)

Extra-adrenal invasion,

not involving other organs--------------13 (100\%)----4 (36\%)

Invasion of other organs-----------13 (100\%)----0 $(0 \%)$

Uninvolved adrenal identified----------11 (85\%)------8 (73\%)

Nature of uninvolved adrenal------------7 (54\%)------8(100\%)

Conclusion: The review of origonal reports showed that there is room for improvement in the documentation of features however it should improve with the introduction of RCPath dataset. Review of slides revealed a good correlation with the original reports. The majority $(92 \%)$ of our cases showed more than three features suggestive of malignant behaviour. We are following up these cases to correlate histological findings with clinical behaviour.
References: 1. Weiss LM, Medeiros LJ, Vickery AL, Jr. Pathologic features of prognostic significance in adrenocortical carcinoma. Am J Surg Pathol 1989;13:202-6.

2. van Slooten H, Schaberg A, Smeenk D, Moolenaar AJ. Morphologic characteristics of benign and malignant adrenocortical tumors. Cancer 1985;55:766-773.

3. Weiss LM. Comparable histologic study of 43 metastasizing and nonmetastasizing adrenocortical tumors. Am J Surg Pathol 1984;8:163-169.

4. Hough AJ, Hollifield JW, Page DL, Hartmann WH. Prognostic factors in adrenal cortical tumours. Am J Clin Pathol 1979;72:390-399.

5.The Royal College of Pathologists, UK. Dataset for histopathology reporting in adrena cortical carcinoma and malignant phaeochromocytoma/paraganglioma. February 2006"

\section{PROTECTIVE REGULATORY T CELL GENERATION IN AUTOIMMUNE DIABETES BY DNA COVACCINATION WITH ISLET ANTIGENS AND A SELECTIVE CTLA-4 LIGAND}

Yelena Glinka, Department of Laboratory Medicine, St. Michael's Hospital, Toronto, Toronto, ON, Canada; Yigang Chang, Department of Pediatrics, University of Pittsburgh, Rangos Research Center, Pittsburg, PA, United States; Gerald Prud homme, Department of Laboratory Medicine, St. Michael`s Hospital, Toronto, Toronto, ON, Canada

Background: DNA vaccination of autoimmune diabetes-prone NOD mice with unmodified target islet antigens, i.e., preproinsulin (PPIns) or glutamic acid decarboxylase 65 (GAD65), is poorly protective.

Design: NOD mice were co-vaccinated with plasmid DNA expressing mutant B7.1 and PPIns-GAD65 fusion protein (Ins-GAD). Protection against diabetes was studied in vivo. Regulatory $\mathrm{T}$ cells $(\mathrm{Tr})$ were sorted from the spleen cell suspension of the vaccinated mice by magnetic sorting and their suppressor potential was studied in the primary culture of diabetogenc spleen cells. They were also phenotyped by Flow cytometry.

Results: In this study, we demonstrate protection against disease by covaccination with a mutant B7-1 molecule (B7-1wa) that binds the negative T-cell regulator CTLA-4 (CD152), but not CD28. Codelivery of plasmids encoding an Ins-GAD fusion construct and B71 wa protected against both insulitis and diabetes. In vitro, the $T$ cells of covaccinated mice had negative responses to both insulin and GAD65, and this was restored by adding blocking antibodies to transforming growth factor beta 1 (TGF $\beta-1$ ), suggesting a role for this cytokine. Adoptive transfer experiments revealed that DNA vaccination generated protective CD4+ Tr of either CD25+ or CD25- phenotype. Furthermore, vaccinated mice had increased numbers of T cells with Tr-associated markers, such as CTLA-4, Foxp3 and membrane-bound TGF- $\beta$. Tr cells inhibited the responses of diabetogenic T cells to islet antigens, and depletion of T cells expressing membrane-bound TGF $\beta-1$ abolished the suppressive effect.

Conclusion: Selective engagement of CTLA-4 during islet-antigen DNA vaccination induces $\operatorname{Tr}$ cells that protect against this autoimmune disease.

\section{LIPID-RICH NEUROENDOCRINE TUMORS OF THE DIGESTIVE} SYSTEM

György Illyés, Semmelweis University, Budapest, Hungary; Csaba Lódi; Attila Kovács, 2nd Department of Pathology, Budapest, Hungary; Tibor Tihanyi; Péter Kupcsulik, 1st Department of Surgery, Budapest, Hungary

Background: Lipid-rich variant endocrine tumors are of special interest because of their differential diagnosis or occurrence in MEN syndromes.

Design: In our records we have found 3 tumors of this type; one primary carcinoma of the liver, one pancreatic tumor and a case of appendiceal carcinoid. We review these cases and present related special histology and outcomes as results.

Results: Case 1.63 years old male patient with a history of hypertension was admitted with jaundice, weight loss and elevated cholestatic liver enzymes. Radiology disclosed a tumor localized in the hilar region of the liver causing biliary obstruction. Owing to the absence of metastases and the patient's good clinical condition total resection of the tumor with partial hepatectomy was performed. The tumor was in close contact with the left hepatic duct, it consisted from an intrahepatic mass measuring $3 \mathrm{~cm}$ in diameter with invasive margins and from a second part of intraluminal growth into the left hepatic duct extending to the choledochus. The left hepatic lobe was atrophic, and there were no lymph node metastases. A malignant neuroendocrine tumor was diagnosed. The patien had an uneventful recovery. No other endocrine tumors were observed. He had undergone chemotherapy and by now has had a more than 2-years tumor free survival. Case 2. 37 years old woman was incidentally diagnosed by ultrasound with a tumor of the head of the pancreas. Partial duodeno-pancreatectomy was performed. A well circumscribed tumor measuring $5 \mathrm{~cm}$ in diameter was found. Microscopically there were no signs for cell proliferation or invasive growth. A well differentiated non-functioning pancreatic endocrine tumor was diagnosed with a probable benign or low malignant potential. She had an uneventful recovery and was referred for von Hippel Lindau syndrome genetic screening. Case 3.67 years old man was operated for acute appendicitis. He had no other complaints and no previous disease or surgery. At the tip of the appendix a yellowish tumor of $2 \mathrm{~cm}$ in diameter was noted. Proximally to this acute inflammation was seen. The tumor had invaded the wall of the appendix to the subserosa. Serotonin was demonstrated by immunohistochemistry. A carcinoid tumor with a highly probable benign biology was diagnosed. The patient has had no subsequent presentations to our clinic. PathologyHistologically all three tumors were lipid-rich neuroendocrine neoplasms. They showed a solid, nesting and pseudo-glandular structure, characterized by differentiated cells with clear cytoplasm due to microvesicular fat accumulation proved by Sudan IV staining. All tumors were PAS negative. Tumor cell nuclei were rounded and partly displaced with endocrine chromatin features. Immunohistochemically, Chromogranin A, Synaptophysin, Neuron Specific Enolase and Cytokeratin 7 reactions were positive. Lipid vacuoles, dense core granules and abundant round mitochondria were seen by electron microscopy. The pancreatic neoplasm was also positive for Vimentin, S-100 and Inhibin.

Conclusion: Lipid-rich neuroendocrine tumors may present in different localizations as either carcinomas or tumors of borderline malignant potential. They show the same 
evolution as other neuroendocrine tumors. Hepatic and appendiceal lipid-rich variants were not published in the literature until now.

\section{FINE NEEDLE ASPIRATION BIOPSY AFTER THYROSTATIC DRUGS AND J131 TREATMENT}

Magdalena Jaworska; Dariusz Lange; Barbara Lange; Dorota Ponikiewska, Institute of Oncology, Gliwice, Poland

Background: Many patients with thyrotoxicosis have been treated with J131 and antithyroid drugs but only a few cytologic observation have been published since several years. The aim of this study was to evaluate the cytological features of smears after treatment changes

Design: 158 cytologic smears from 83 patients suffered from toxic goiter and 38 with Graves' disease were evaluated. Patients were divided into three groups according to the manner of treatment. 58 patients $(48 \%)$ were treated with antithyroid drugs as well as J131. Another group of 45 patients $(37 \%)$ were treated exclusively with antithyroid drugs. The third group of 18 patients $(15 \%)$ was managed by radioactive iodinetherapy without antithyroid drugs. All ultrasound guided fine needle biopsies were performed by pathologist. The smears were examined for cellularity, pattern of follicular cells, nuclear size, chromatin texture, presence of histiocytes, fibrosis and amount of colloid. The smears with normal thyrocytes in groups, isolated, in follicular pattern, with histiocytes, fibrosis and colloid, were reported as "nodular goiter". Sparse number of follicular cells was signed as "low cellularity". The smears abounded of the colloid and histiocytes, devoid of follicular cells were reported as "colloid". Mild nuclear changes as anisonucleosis, hyperchromasia, macronucleosis were signed as "polymorphism". High cellularity, follicular pattern, monolayer tissue fragments were reported as "hyperplastic nodule".

Results: The most frequent smears were reported as "low cellularity" and "nodular goiter" in all three groups of patients. Two smears suspected of papillary carcinoma were confirmed in histologic specimen after surgery. There was one false negative diagnosis of fine needle biopsy in patient with papillary carcinoma found in histologic specimen. In this case the cytologic material contained one group of 4 follicular cells with slightly enlarged nuclei and smudged chromatin and another 1 group of 5 normal thyrocytes and was reported as low cellularity.

Conclusion: The most frequent features observed in smears obtained in aspiration biopsy from patients treated for thyrotoxicosis was low cellularity. In spite of their low diagnostic value, this observation may be concerned with fibrosis, frequent after J131 treatment.

\section{DIAGNOSTIC UTILITIES OF KNOWN MOLECULAR MARKERS IN HASHIMOTO'S THYROIDITIS ASSOCIATED PAPILLARY CARCINOMA}

Soojin Jung; Hye-kyoung Yoon, Inje University, College of Medicine, Busan Paik Hospital, Busan, Korea, South

Background: Papillary thyroid carcinoma (PTC)-like nuclear alterations may be noted in almost one-third of Hashimoto's thyroiditis (HT) on routine microscopic examination. Moreover, increased incidence of PTC associated with HT has been reported. Sometimes, we are confronted with diagnostic difficulty in case of HT with follicles showing equivocal PTC-like nuclear changes. This study was aimed to determine molecular expressions in follicular cell of HT associated with PTC and to evaluate the utility of these molecular markers to distinct equivocal PTC-like nuclear features and true PTC.

Design: Both area showing PTC-like nuclear features in HT and area of true PTC were selected in each 56 cases of HT associated with PTC. Immunohistochmical stainings for known molecular markers of papillary carcinoma (HBME-1, GAL-3, CK19, RET) were performed on five tissue microarray blocks consisted of 112 cores

Results: The expression rates of HBME-1, GAL-3, RET, CK19 were 25.0\%, 44.6\%, $87.5 \%, 83.9 \%$ in HT area and $92.8 \%, 80.4 \%, 89.3 \%, 91.1 \%$ in PTC area, respectively. The specificities (number of negative cases out of total number of HT) of these marker were $75.0 \%, 55.3 \%, 12.5 \%, 16.1 \%$ in HBME-1, GAL-3, RET, CK19. HBME- 1 was highly expressed in PTC and its specificity for differentiation PTC from PTC like feature was the highest. Both positive and negative predictive values of HBME-1 were $78.79 \%$ and $91.30 \%$, the highest among four markers.

Conclusion: Diffuse expression of molecular markers in PTC-like follicular cells in HT suggest the possibility of early premalignant transformation in HT. And HBME-1 was useful marker in practically distinction of follicular cells with PTC-like nuclear features from true PTC.

\section{EXPRESSION OF ID-1 AND ID-2 IN HYPERPLASTIC THYROID TISSUE AND THYROID CARCINOMA}

Ji Eun Kim; Young A Kim, Seoul National University Boramae Hospital, Seoul, Korea, South; Sungshin Park, Dongkuk University Hospital, Seoul, Korea, South

Background: Id proteins are a family of helix-loop-helix proteins and are regarded to be negative regulators of cell differentiation. In general, Id-1 and Id-2 expressions are upregulated during tumor development and progression in a variety of neoplasms, and these expressions may be associated with aggressive tumor behavior. However, little is known about the roles of Id- 1 and Id-2 in thyroid neoplasms.

Design: The expressions of Id-1 and Id-2 were assessed immunohistochemically in 310 normal, hyperplastic, and neoplastic thyroid tissues using tissue microarrays.

Results: Normal thyroid tissues rarely expressed Id-1 or Id-2. Moreover, whilst Id-1 expression was more elevated in malignant thyroid tissue than in hyperplastic thyroid tissue, Id-2 expression was more variable. No significant differences were observed between histologic subtypes of thyroid carcinomas with respect to Id-1 or Id-2 expression. Follicular adenomas showed higher expressions of Id-1 and Id-2 than thyroid carcinomas, but no significant association was found between clinicopathological parameters and Id1 expression, though Id-2 expression was significantly reduced in metastatic, stage IV tumors.

Conclusion: The expressions of Id-1 and Id-2 were elevated in hyperplastic and neoplastic thyroid tissues. However, neither appears suitable as a marker of malignancy or an aggressive phenotype, although Id-2 expression in advanced thyroid carcinomas may reflect a favorable prognosis.

213 DIVERGENT EXPRESSION AND ACTION OF FIBROBLAST GROWTH FACTOR RECEPTORS (FGFRS) 1 AND 2 IN THYROID CANCER

Tetsuo Kondo, Department of Pathology, University Health Network, Toronto, ON, Canada; Shereen Ezzat, Mount Sinai Hospital and University of Toronto, Toronto, ON, Canada; Daniel Winer; Sylvia L Asa, Department of Pathology, University Health Network, Toronto, ON, Canada

Background: The fibroblast growth factor (FGF) family of ligands plays fundamental roles in development and the tumorigenetic process. The cellular effects of FGFs are mediated by FGF receptors (FGFRs 1-4) in mammals. The prototypic FGFR consists of three extracellular immunoglobulin (Ig)-like domains, a single transmembrane domain, and an intracellular tyrosine kinase domain. A major alternative splicing occurs within the first Ig-like domain of FGFRs 1-3, resulting in "b" and "c" receptor isoforms that exhibit distinct ligand binding specificities. The individual and combined functions of different members of the FGFR family in thyroid cancer progression remain unknown.

Design: In this study we focused on the expression of the two principal members of the FGFR family (1 and 2) that transduces fibroblast growth factor (FGF) signals in human thyroid tissue and tumor cell lines. We used siRNA-mediated downregulation and CMV promoter mediated over-expression of FGFRs to determine the individual or combined impact of FGFR1 \& 2 on cancer cell proliferation and growth in xenografted SCID mice. Results: FGFR1 knockdown in thyroid carcinoma WRO cell line resulted in decelerated S-phase entry and tumor growth suppression in xenografted SCID mice. On the other restoration of the FGFR2b isoform interrupted BRAF phosphorylation and consequently ERK1/2 in WRO cells. Moreover, xenograft tumor volume was reduced in FGFR2b expression WRO cells compared with FGFR2b negative control cells.

Conclusion: These findings suggest a unique FGFR isoform profile in thyroid cancer with a predominantly anti-tumor differentiating effect assigned to the FGFR2b isoform. In contrast, FGFR1 appears to mediate a proliferative signaling cascade. These findings underscore the complex network of this family of tyrosine kinases in modulating cancer cell growth and predict the need for highly selective inhibitors in the control of disease progression.

214 BRAF VK600-1E DEL AND BRAF K601E MUTATIONS IN A CASE OF MULTICENTRIC PAPILLARY THYROID CARCINOMA WITH RETROSTERNAL METASTASIS

Cristina Manieli; Maria Letizia Lai; Sara Pillai; Pierpaolo Coni; Giuseppina Parodo Gavino Faa, University of Cagliari, Cagliari, Sardinia, Italy

Background: Papillary carcinoma (PTC) is the most common malignancy in thyroid gland. Multiple foci of PTC are frequently found in the same gland. Molecular studies for RET/PTC rearrangements, demonstrated the frequent independent onset of these foci. PTC is frequently associated with BRAF gene mutations. BRAFV600E accounts for more than $80 \%$ of all the BRAF mutations and is associated with PTCs which display a papillary growth pattern. This mutation has been also associated with a poor prognosis. Another BRAF mutation, the BRAF K601E, has been found in about $10 \%$ of follicular variantPTCs. Recently, a case of an incidental $5 \mathrm{~mm}$ in diameter micro-PTC (solid variant) with a new BRAF mutation (BRAF VK600-1E del) has been reported.

Design: we describe a 61 year old lady with two histological different PTCs in the thyroid gland, carrying two different BRAF mutations. Genomic DNA was extracted from the paraffin-embedded thyroid tumors and from the metastasis. The exon 15 of the BRAF gene was amplified by PCR using direct sequencing of specific amplicon. We also used the Hinf I digestion followed by southern hybridization (Hinf I-RFLP test)

Results: Macroscopic examination showed a $149 \mathrm{~g}$ gland, with an infiltrating nodule, $70 \mathrm{~mm}$ in diameter, with extraglandular extension, in the left lobe and a capsulated nodule, $40 \mathrm{~mm}$ in diameter, in the right lobe. In the upper-mediastinal region a $50 \mathrm{~mm}$ in diameter metastasis, infiltrating the sternal bone, was removed. The histological study revealed the typical features of Tall cell-PTC in the left lobe lesion and in the mediastinal metastasis; the nodular lesion in the right lobe was classified as a follicular variant-PTC. BRAF VK6001E deletion was detected in the Tall-cell-PTC and in its mediastinal metastasis. It was more frequent in the mediastinal metastasis cells rather than in primary tumor cells. BRAF K601E mutation was detected in the follicular variant-PTC of the right lobe and not in the metastasis. Immunohistochemical studies showed a high Ki67 proliferative index both in the Tall cell-PTC and in its metastasis; on the contrary a very low Ki67 proliferative index was found in the follicular variant-PTC nodule.

Conclusions: To date, multiple mutations of BRAF oncogene in multicentric PTC, have not been described. In the present study, we report the detection of two different BRAF mutations, BRAFVK601-1E and BRAF K601E in two PTCs foci, with different histological pattern, in the same gland. This date confirm the different clonal origin of the two neoplastic nodules. The BRAF VK601-1E seems to be associated with the metastatizing potential, in contrast with the previously low grade published case with this mutation.

215 ANTI-TUMOR EFFECTS OF TEMOZOLOMIDE IN A MAN WITH LARGE, INVASIVE PROLACTIN SECRETING PITUITARY NEOPLASM

Fateme Salehi, Department of Laboratory Medicine, St. Michael's Hospital, University of Toronto, Toronto, ON, Canada; Luis V. Syro, Department of Neurosurgery, Hospital Pablo Tobon Uribe and Clinica Medellin, Medellin, Colombia; Humberto Uribe, Department of Neurosurgery, Clinica SOMA, Medellin, Colombia; Luis C Penagos, Division of Otolaryngology, Clinica Medellin, Medellin, Colombia; Leon D. Ortiz, Section of Neurooncology, Instituto de Cancerologia, Clinica Las Americas, Medellin, Colombia; Camilo E Fadul, Sections of Hematology/Oncology and Neurology, Darmouth Hitchcock Medical Center, Lebanon, NH, United States; Eva Horvath; Kalman Kovacs, Department 
of Laboratory Medicine, St. Michael's Hospital, University of Toronto, Toronto, ON, Canada

Background:Temozolomide, an amidazo-tetrazine derivative, is an alkylating with antitumor properties against gliomas and other types of neoplasms. It is rapidly absorbed following oral intake, and is readily converted to its active metabolite at physiologic $\mathrm{pH}$. Temozolomide crosses the blood brain barrier with ease and it was introduced to treat patients with neoplasms of the central nervous system, melanomas metastatic to the brain, as well as various neuroendocrine tumors. To date, the effect of temozolomide was reported in three patients with pituitary carcinoma. Here, we report clinical, laboratory, histological, immunohistochemical, and electron microscopic findings following temozolomide therapy in a patient with prolactin secreting pituitary adenoma.

Design: A 46 year old man with a large invasive prolactin secreting pituitary neoplasm was treated with temozolomide after repeated surgeries, irradiation, and dopamine agonist medication failed to induce permanent clinical and laboratory improvement. In 1989, frontal craniotomy was performed to resect the pituitary tumor, followed by irradiation. After recurrence in June 2002, the tumor was partially removed. Seven months later, the patient presented with visual disturbances and hypopituitarism; blood FSH, LH, and cortisol levels were low. Markedly elevated serum prolactin levels were evident (1885 $\mathrm{ng} / \mathrm{ml}$, normal $<11)$ and a sellar lesion $(47 \times 44 \times 37 \mathrm{~mm})$ was revealed by MRI. The tumor was partially resected by transsphenoidal surgery and the patient was treated with hormone replacement. Blood prolactin levels remained elevated after surgery. Bromocriptine and later Cabergoline were administered with no response. Blood prolactin levels continued to rise during the next few months, and MRI revealed a large tumor $(35 \times 30 \times 30 \mathrm{~mm})$, prompting another resection in May 2004. Although blood prolactin levels decreased postoperatively to $156 \mathrm{ng} / \mathrm{ml}$, they remained above normal levels. The tumor continued to grow $(50 \times 45 \times 50 \mathrm{~mm})$. In October 2004, only a small portion of the tumor was removed due to its hard consistency, and blood prolactin levels remained very high. In January 2005 , temozolomide administration was started at a dose of $200 \mathrm{mgm} 2 /$ day x 5 days, repeated every 28 days for 7 months.

Results: Following temozolomide therapy, the patient's clinical symptoms improved, and blood prolactin levels decreased to $30 \mathrm{ng} / \mathrm{ml}$ and the MRI demonstrated hemorrhage and necrosis within the tumor. In October 2005, the tumor, which now had a soft consistency, was resected by surgery. Histological, immunohistochemical and electron microscopic investigation showed major differences in pre- and post-treated tumor. In contrast to the tumor not exposed to temozolomide, after temozolomide administration the tumor was more differentiated, consisting of larger cells. Hemorrhagic and necrotic areas, focal fibrosis and neuronal transformation were observed. In addition, a decreased number of mitotic figures were evident and the Ki-67 nuclear labeling index was significantly lower indicating reduced cell proliferation.

Conclusion: Temozolomide treatment of a patient with a large, rapidly growing, invasive prolactin secreting pituitary neoplasm resulted in marked clinical and laboratory improvement. Although no definitive conclusions can be drawn based on one single case, our promising findings warrant recommendation of temozolomide therapy in patients with aggressive prolactin secreting pituitary neoplasms.

\section{E-CADHERIN AND $\beta$-CATENIN EXPRESSION IN PANCREATIC ENDOCRINE TUMORS}

Stefano Serra, University Health Network and Toronto Medical Laboratories, Toronto, ON, Canada; Sima Salahshor, Department of Medical Biophysics, Ontario Cancer Institute, Toronto, ON, Canada; Sylvia L Asa; Runjan Chetty, University Health Network and Toronto Medical Laboratories, Toronto, ON, Canada

Background: decrease in E-cadherin is considered a molecular event in dysfunction of the cell-cell adhesion system, triggering invasion and metastasis in many malignancies, including those of endocrine origin. In addition, alterations in the cadherin-catenin system may also be involved in tumorigenesis. E-Cadherin and $\beta$-Catenin, components of the Wnt signal transduction pathway, may serve as a common switch in central processes that regulate cellular differentiation and growth.

Design: to examine if abnormalities of the Wnt signaling pathway occur in Pancreatic Endocrine Tumors (PETs) and correlate with clinical-pathological parameters. Fifty-one cases were retrieved for this study from the archives. Tissue microarray construction: for each case, 4 to 14 cores measuring $1.0 \mathrm{~mm}$ each were drilled from the blocks. Tissue cores from normal pancreas were used as controls and for orientation purposes. Size of tumor, presence or absences of necrosis, gross invasiveness/demarcation, lymphovascular invasion, and lymph node involvement and liver metastasis were recorded. The mitotic count, expressed per $50 \mathrm{HPF}$ and MIB-1 index of the entire tumor were assessed. All the TMA blocks were stained with an extensive panel of endocrine markers as well as ECadherin, $\beta$-catenin APC and GSK-3 $\beta$.

Results: 24 were male patients and 27 female, ranging in age from 23 to 80 years (mean 49.8 years). Seven patients had MEN1 syndrome and 1 von Hippel Lindau disease. The remaining cases were classified as sporadic PETs. The tumors ranged in size from $0.8 \mathrm{~cm}$ to $8.7 \mathrm{~cm}$ with a mean size of $3.6 \mathrm{~cm}$. The size was unknown in 6 cases. Ten patients had lymph node spread and a further 6 had liver metastasis. Membranous immunoreactivity for E-Cadherin/ $\beta$-Catenin was considered normal, any reduction or loss of this pattern of immunoreactivity and/or nuclear, cytoplasmic staining was considered as abnormal. Accordingly, PETs were divided into 4 groups. 23/51 (45\%) cases showed the typical membranous immunoreactivity for both E-Cadherin/ $\beta$-Catenin: 14 were confined to the pancreas, 6 were locally invasive and 3 showed lymph nodal invasion, but none had liver metastasis. 21/51 cases showed an abnormal $\beta$-Catenin expression. 19/21 (37\%) cases also showed E-Cadherin abnormalities. Of these, 8 were confined to the pancreas, 2 were locally invasive, 4 showed lymph nodal and 5 liver involvement. Seven cases had only E-Cadherin abnormalities: 3 were non-invasive PETs, 2 locally invasive and 3 with lymph node involvement. One $\beta$-Catenin abnormal/E-Cadherin normal PET had hepatic metastasis. Of cases with E-Cadherin abnormalities, 3 showed loss of membranous $\beta$-Catenin (2 confined to the pancreas and 1 with lymph node involvement). All fifty-one cases showed cytoplasmic immunoreactivity for APC, and cytoplasmic and nuclear positivity for GSK$3 \beta$. APC and GSK-3 $\beta$ did not show any correlation with $\beta$-Catenin or E-Cadherin. Conclusion: Abnormalities of $\beta$-Catenin and/or E-Cadherin are seen in the majority of PETs and may identify a subgroup of more aggressive tumors; those more frequently associated with lymph node and hepatic involvement and may be involved in early tumorigenesis of PETs. Alterations of E-Cadherin are more frequently seen than those of $\beta$-Catenin.

\section{P53 DOES NOT PLAY A ROLE IN DETERMINING PROGNOSIS IN} PANCREATIC ENDOCRINE TUMORS

Stefano Serra, University Health Network/Toronto Medical Laboratories, Toronto, ON, Canada; Sima Salahshor, Department of Medical Biophysics, Ontario Cancer Institute, Toronto, ON, Canada; Sylvia L Asa; Runjan Chetty, University Health Network and Toronto Medical Laboratories, Toronto, ON, Canada

Background: p53, a tumor suppressor gene, is involved in the pathogenesis of numerous of neoplasms, and p53 protein overexpression has been considered a prognostic marker Dysregulation of the Wnt signaling pathway, with abnormalities of $\beta$-Catenin and ECadherin, is also considered important in the tumorigenesis and outcome of neoplasms.

Design: To examine the relationship between $\mathrm{p} 53$ and $\beta$-Catenin/E-Cadherin immunoexpression, and correlate with clinicopathological features of Pancreatic Endocrine Tumors (PETs). Fifty-one cases were retrieved for this study from the archives. Tissue microarray construction: for each case, 4 to 14 cores measuring $1.0 \mathrm{~mm}$ each were drilled from the blocks. Tissue cores from normal pancreas were used as controls and for orientation purposes. Size of tumor, presence or absences of necrosis, gross invasiveness/ demarcation, lymphovascular invasion, and lymph node involvement and liver metastasis were recorded. The mitotic count, expressed per 50 HPF and MIB-1 index of the entire tumor were assessed. All the TMA blocks were stained with an extensive panel of endocrine markers as well as p53, $\beta$-Catenin and E-Cadherin. p53 staining was assessed as follow: negative if $\leq 5 \%$ tumor cell immunolabeling, $1+: 6-25 \%, 2+: 26-50 \%, 3+$ : $51-75 \%, 4+:>75 \%$

Results: 24 were male patients and 27 female ranging in age from 23 to 80 years (mean 49.8 years). Seven patients had MEN1 syndrome and 1 von Hippel Lindau disease. The remaining cases were classified as sporadic PETs. The tumors ranged in size from $0.8 \mathrm{~cm}$ to $8.7 \mathrm{~cm}$ with a mean size of $3.6 \mathrm{~cm}$. The size was not known in 6 cases. Ten patients had lymph node spread and a further 6 had liver metastasis. 21/51 (41\%) cases showed p53 overexpression with the majority of cases being +1 and $+2.28 / 51(55 \%)$ showed abnormalities of $\beta$-Catenin/E-Cadherin expression. Eleven of the $21 \mathrm{p} 53+$ cases lacked any abnormality of $\beta$-Catenin/E-Cadherin. Of these, 8 were confined to the pancreas and 3 showed local invasion. Ten of the $21 \mathrm{p} 53+$ tumors had $\beta$-Catenin/E-Cadherin abnormalities Of these 5 were confined to the pancreas, 1 was locally invasive, 3 cases had lymph node metastases, 1 case liver metastasis. Twelve of the 30 p53- cases had normal expression $\beta$-Catenin/E-Cadherin. Six of these cases were confined to the pancreas, 3 showed loca invasion and the remaining 3 cases, lymph node involvement. Of the eighteen p53-cases with abnormalities of $\beta$-Catenin/E-Cadherin; 6 were confined to the pancreas, 3 cases had local invasion, 4 lymph node and 5 liver metastases.

Conclusion: $41 \%$ of the PETs studied showed overexpression of $\mathrm{p} 53$, while abnormalities of $\beta$-Catenin/E-Cadherin were present in $55 \%$ of cases. Overexpression of p53 alone without abnormality of $\beta$-Catenin/E-Cadherin appeared to be an early event in the genesis of PETs, as p53 overexpression was seen in pancreas-confined tumors without lymph node and liver spread. Metastases were found in both p53+ and p53- PETs. However, cases with abnormality of $\beta$-Catenin/E-Cadherin frequently spread to lymph node and hepatic metastases, indicating a more aggressive outcome. $\beta$-Catenin/E-Cadherin were better determinants of behavior in both p53 + and p53- cases rather than p53 on its own.

218 THE SPECTRUM OF LIPOMATOSIS IN NEOPLASTIC AND NONNEOPLASTIC FOLLICULAR THYROID LESIONS

Lynette Sholl; Vânia Nosé, Brigham and Women`s Hospital, Boston, MA, United States

Background: There is little published data regarding the presence of fat within neoplastic and nonneoplastic thyroid conditions. Isolated case reports describe hamartomatous adiposity, adenolipomas, and fat infiltration in diverse lesions. The largest published series of 17 cases described lipomatous change within papillary thyroid carcinoma, benign and malignant follicular lesions, and a range of nonneoplastic conditions. Metabolic diseases involving the thyroid are very rare; diseases involving lipid metabolism are not reported, with a single report describing age-related fat accumulation in follicular cells. The significance of fat in these processes is unknown.

Design: From a total of 373 follicular nodules diagnosed at Brigham and Women's Hospital from 2000-2006, we examined 79 follicular adenomas (17 of oncocytic type), 12 follicular carcinomas, 8 oncocytic cell carcinomas, and 42 adenomatous nodules. In neoplastic lesions, lipomatous change was defined as the presence of fat admixed with neoplastic follicular cells; in benign lesions, lipomatous change was defined as fat admixed with follicles away from interlobular fibrovascular septae and away from the edges of the gland. Follicular neoplasms undergoing degenerative changes with associated foamy macrophages were excluded.

Results: We identified a total of 41 lesions containing lipomatous change: 6 (of 17) follicular adenomas of oncocytic type, 8 ( of 62) follicular adenomas, 1 (of 8) follicular carcinomas of oncocytic type, 0 (of 12) follicular carcinomas, and 9 (of 42) adenomatous nodules. The median age of patients with lipomatous thyroid nodules was 53 years (range 30- 78) and with nonlipomatous nodules was 50 years (range 14- 89). Fatty metaplasia in the form of lipidization of neoplastic follicular cells was more likely to occur in oncocytic-type follicular adenomas than in follicular adenomas of usual type $(p=0.042)$. Adenomatous nodules arising in the background of multinodular hyperplasia were significantly more likely to contain fat ( 6 of 15) than those arising singly or in association with other lesions ( 3 of 27$)(p=0.038)$. Adipose cells within adenomatous nodules were $S-100$ positive and 
TTF-1 negative, suggestive of stromal cell metaplasia. The presence of lipomatous change did not correlate with clinical hyper- or hypothyroidism nor with patient sex. Although benign neoplasms contained fatty metaplasia (14 of 79) more frequently than did malignant neoplasms ( 1 of 20$)$, this was not statistically significant $(\mathrm{p}=0.14)$.

Conclusion: Our findings suggest that there are two possible pathways for lipomatous change within thyroid nodules. Some oncocytic-type follicular adenomas demonstrate focal lipidization of tumor cells in association with areas of mature intratumoral fat, suggesting that follicular tumor cells, not stromal cells, are undergoing fatty metaplasia. This is in contrast to our observations of "true" adipose tissue deposition in the stroma of adenomatous nodules. The increased incidence of lipomatous change in oncocytic neoplasms and in adenomatous nodules arising in multinodular hyperplasia is a novel observation and may be related to altered metabolic activity in these lesions. Additional studies are warranted to establish a molecular basis for these observations.

\section{GALECTIN-3: A USEFUL MARKER IN DISTINGUISHING SILENT CORTICOTROPH ADENOMAS OF THE PITUITARY GLAND}

Eleni Thodou; Theodore Argyrakos; George Kontogeorgos, G. Gennimatas Athens General Hospital, Athens, Hellas, Athens, Greece

Background: Functioning corticotroph adenomas are indistinguishable from silent ones. Particularly, silent corticotroph adenomas type 1 are morphologically identical to their functioning counterparts in terms of their histology, histochemistry, immunohistochemistry and ultrastructure. No specific markers to separate them are available so far. Therefore, the necessity for a specific marker is crucial. Galectin-3 (Gal-3) belongs to the family of carbohydrate-binding proteins with high affinity for b-galactoside and it is involved in many biological processes including cell growth and differentiation, cell adhesion, tumor progression, apoptosis and metastasis.

Design: We studied by immunohistochemistry the expression of Gal-3 in $30 \mathrm{ACTH}$ positive corticotroph pituitary adenomas ( 19 functioning and 11 silent) using a monoclonal antibody specific for Gal-3. All functioning adenomas were removed from patients associated with Cushing's disease. The independent variables t-test was used for comparing the mean percentages of Gal-3 in the two different subgroups.

Results: Eighteen of the functioning corticotroph adenomas (94.73\%) expressed Gal3 with diffuse cytoplasmic reactivity and focal tendency to membranous enhancement. The mean percentage of Gal-3 positive adenoma cells was $77 \%$. Among the silent corticotroph adenomas, $9(81.81 \%)$ were negative, while the remaining two showed Gal-3 immunoreactivity in $15 \%$ and $80 \%$ of their adenoma cell population (mean percentage: $8.72 \%)$. The statistical differences between functioning and silent adenomas were highly significant $(\mathrm{p}=0.001)$.

Conclusion: Gal-3 is highly expressed in functioning corticotroph adenomas whereas, the substantial majority of silent adenomas are negative for Gal-3. These observations indicate that Gal-3 may serve as a useful marker in distinguishing silent corticotroph adenomas of the pituitary gland.

\section{CORRELATION OF HISTOPATHOLOGY USING THE PASS SYSTEM AND PROLIFERATIVE INDEX IN PHEOCHROMOCYTOMAS AND APPLICATION TO EXTRA-ADRENAL PARAGANGLIOMAS}

David Wu; Elizabeth A. Bundock; Vânia Nosé, Brigham and Women's Hospital, Boston, MA, United States

Background: Adrenal pheochromocytomas are challenging lesions to morphologically classify into those with likely malignant versus benign clinical behavior in a prospective fashion. A scoring system, designated Pheochromocytoma Adrenal Scaled Score, PASS, was recently proposed to aid in this categorization effort. Thus far, no validation studies have been performed assessing the robustness of this system, especially in correlating morphological parameters with tumor proliferative index, as assessed by MIB-1/Ki-67 staining, which has been most consistently correlated with malignancy in independent studies. In the original report, staining for MIB-1 was remarkably variable, ranging from $0-52 \%$ of nuclei, and did not correlate with adverse clinical outcome. We sought to further compare MIB-1 staining versus PASS scores in a cohort of adrenal pheochromocytomas using a tissue microarray platform in consideration of this proposed scoring system. In addition, we evaluated the application of this scoring system to extra-adrenal paragangliomas, as these lesions likely result from similar underlying pathobiological mechanisms.

Design: Sixty-six adrenal pheochromocytomas and 61 extra-adrenal paragangliomas from patients of $42.6 \pm 17.4$ years mean age $(44 \%$ male) were retrieved from the archive files of the Departments of Pathology of Brigham and Women's Hospital and Children's Hospital, Boston, MA from the period of 1981 to 2005, and included primary and recurrent/metastatic lesions in both syndromic and sporadic patients. A representative slide of each case was reviewed to score PASS, with three representative areas selected for tissue-microarray analysis. MIB-1 staining intensity was subsequently categorized as high, moderate, weak, or none and the number of nuclei stained per unit area was quantified. The number of MIB1 staining nuclei was then correlated with PASS score as a function of staining intensity. Results: Our data reveal all adrenal pheochromocytomas studied had a PASS score greater than 4 , suggesting potential biologically aggressive behavior, according to the proposed scoring system. Of note, there was a broad range of PASS scores for which there was weak to no MIB-1 staining, including cases with high PASS scores. In contrast, several cases with lower PASS scores showed relatively increased MIB-1 staining. Extension and application of the PASS to extra-adrenal paragangliomas demonstrated similar variability, showing no- to weak staining in higher PASS score cases and increased staining in some lower PASS scores.

Conclusion: Prospective implementation of the PASS likely results in a high false positive rate with low positive predictive value, as all of our cases were designated as being histologically malignant using PASS. Correlation of PASS with MIB-1 showed significant variability in this cohort, similar to the original findings of the PASS. Taken together, we caution that additional information and insight is needed before widespread adaptation of this scoring method, and suggest that more discriminative parameters, including possibly genetic-molecular markers, be considered for optimal stratification of benign versus malignant potential.

\section{Environmental}

221 ALTERATIONS OF CELL CYCLE REGULATORS IN CONVENTIONAL RENAL-CELL CARCINOMAS AFTER THE CHERNOBYL ACCIDENT. COMPARISON WITH SPANISH CASES

Antonio Llombart-Bosch, Department of Pathology, Medical School, University of Valencia, Valencia, Spain; Alina Romanenko, Department of Pathology, Academy of Medical Sciences of Ukraine, Kiev, Ukraine; Luisa Morell-Quadreny, Department of Pathology, Medical School, University of Valencia, Valencia, Spain; José Antonio LópezGuerrero, Unit of Molecular Biology. Fundación Instituto Valenciano de Oncología, Valencia, Spain; David Ramos, Department of Pathology, University of Valencia, Valencia Spain; Alexander Vozianov, Department of Urology, Academy of Medical Sciences of Ukraine, Kiev, Ukraine

Background: There is hypothesized evidence that chronic long-term, low-dose ionising radiation (IR) effects cell cycle regulatory molecules and influences renal-cell carcinogenesis.

Design: The aim of our study was to assess by immunohistochemistry (IHC), using a tissue microarray technique, p53, mdm2, p21WAF1/CIP1, p16INK4a, p14ARF and Ki-67 protein expression in 78 conventional renal cell carcinomas (cRCCs) from Ukrainian patients with different degrees of radiation exposure after the Chernobyl accident, in comparison with analogue Spanish tumors.

Results: Highly significant differences between the Ukrainian and Spanish groups were found in the elevated levels of p53, Ki-67, p21WAF1/CIP1, cyclin D1 and especially p14ARF expression with no relationships with tumor stage or nuclear grade.

Conclusion: Our present findings suggest that chronic long-term, low-dose IR exposure leads to activation and alteration of both $\mathrm{p} 53 / \mathrm{mdm} 2 / \mathrm{p} 21 \mathrm{WAF} 1 / \mathrm{CIP} 1$ protein expression as well as $\mathrm{p} 16 \mathrm{INK} 4 \mathrm{a} / \mathrm{p} 14 \mathrm{ARF}$ locus proteins which could lead to disruptions and loss of cell cycle checkpoints and, thereby, to enhanced tumor progression and aggressivity.

\section{CANCER: AN EMERGING HEALTH PROBLEM IN NORTH CENTRAL} NIGERIA

Barnabas Mafala Mandong, Jos University Teaching Hospital, Jos, Nigeria

Background: Cancer as non-communicable disease is now emerging as a major public health problem in the tropics competing with infections like tuberclosis, malaria, HIV and others. There are no complete data on cancer statistics in most developing countries of the tropics.

Design: A retrospective review of cancer daignoses from the cancer registry of regional histopathology laboratory collected over twenty years [1985-2004] at Jos University teaching hospital, Jos.

Results: There were a total of 5606 cancers diagnosed between 1985 and 2004. This figure represents $14.6 \%$ of all diagnosed specimens within the same period. There were 3503 cases of cancer in females and 2103 in males giving a 1:0.6 ratio. Contributary factors to high mortality were poverty,with most people living below a dollar per day, and a high cos of screening for cancers, for example that of cervical cancer is between $\$ 100-\$ 150$.

Conclusion: Cancer which was believed to be rare in north central Nigeria is now a major public health problem competing with infectious diseases.

\section{TOXIC ELEMENTS, ENVIRONMENTAL EXPOSURE IN CEARA,} BRAZIL

Dary Oliveira, Federal University of Ceara, Fortaleza, Brazil

Background: Introduction of the industrial process of development exposes populations to toxic elements and this will be progressively an important public health problem. Aims of this study are to identify the presence of environmental toxic elements: Al (Aluminum), As (Arsenic), Be (Beryllium), Cd (Cadmium), $\mathrm{Hg}$ (Mercury) and $\mathrm{Pb}$ (Lead) in a population from Ceara, a northeastern state of Brazil.

Design: Methods include coupled mass spectrometry (ICP-MS) used for the determination of toxic elements in scalp hair samples from a population group $(\mathrm{N}=2000)$ over 30 years old and under 80 years. The reference intervals for normal parameters were calculated by Miekeley et al, PUC-Rio, 1998.

Results: Results were considered as toxic levels when more than two standard deviations above mean in parts per million (ppm). Al was found in 38 per cent of the patients, 27 per cent presented $\mathrm{Pb}, 22$ per cent revealed $\mathrm{Hg}, 11$ per cent presented $\mathrm{Cd}$, in 5 per cent we detected $\mathrm{As}$ and $\mathrm{Be}$ was present in 4 per cent of the patients investigated.

Conclusion: This study reveals that the environmental exposure of toxic elements may be more than we could imagine in the general population. The health effects of such chronic, high level exposures are unknown and the influence of this in other disease raises good questions for further study.

224 PATHOLOGICAL STUDIES OF ALL HUMAN AUTOPSY CASES CONCERNED WITH MINAMATA DISEASE IN JAPAN COMPARED WITH THOSE OF OTHER COUNTRIES UNTIL 2005

Akira Yasutake, National Institute for Minamata Disease, Minamata, Japan; Hitosh Takahashi; Akiyoshi Kakita, Brain Research Institute, Niigata University, Niigata, Japan Masumi Sawada, National Institute for Minamata Disease, Minamata, Kumamoto, Japan Hidehiro Tokunaga, Kumamoto University School of Medicine Kumamoto, Kumamoto, Japan

Background: Minamata disease (MD), a classic example of pollution-related health damage in Japan, was first discovered in 1956 around Minamata Bay in Kumamoto 